\title{
Oxygen pressure-dependent control of carbonic anhydrase synthesis in chick embryonic erythrocytes
}

\author{
D. MILLION, P. ZILLNER, AND R. BAUMANN \\ Physiologisches Institut, Universität Regensburg, 8400 Regensburg, Germany
}

Million, D., P. Zillner, and R. BaumanN. Oxygen pressure-dependent control of carbonic anhydrase synthesis in chick embryonic erythrocytes. Am. J. Physiol. 261 (Regulatory Integrative Comp. Physiol. 30): R1188-R1196, 1991.-During chick embryonic development carbonic anhydrase (CA) expression of erythrocytes is kept at a very low level until the last week of incubation (i.e., up to day 14). We have previously obtained evidence that hypoxia is the physiological stimulus for rapid onset of CA synthesis before hatching. Looking for putative signals we have carried out in vitro incubations of embryonic erythrocytes, screening a large number of hormones and second messengers, which were all ineffective, with the exception of the $\mathrm{A}_{1}$ agonist $N^{6}$-phenylisopropyladenosine (adenosine had no effect). However, incubation with embryonic plasma (10\%) from embryos $>6$ days caused a 10-fold increase of the CA activity during $24 \mathrm{~h}$. This increase was not observed when the incubation was carried out with the addition of actinomycin $\mathrm{D}$, cycloheximide, aluminum fluoride, pertussis toxin, or heatinactivated plasma. Mammalian plasma had no effect on CA activity. Filtration experiments show that the molecular mass of the factor is $<2,000 \mathrm{Da}$. We conclude that embryonic plasma contains a heat-labile factor which stimulates CA synthesis via activation of transcription and whose receptor is coupled to a pertussis toxin-sensitive $G$ protein. In vivo the action of the plasma factor is suppressed as long as blood $\mathrm{Po}_{2}$ is high, suggesting the presence of an inhibitor molecule whose synthesis is controlled by the $\mathrm{PO}_{2}$.

embryonic plasma; pertussis toxin; actinomycin D; $N^{6}$-phenylisopropyladenosine; phorbol 12-myristate 13-acetate

CARBONIC ANHYDRASE (CA) is one of the key marker enzymes for erythrocyte differentiation. Erythrocytes from chicks contain only CA type II. It has been shown that, during ontogeny of the chick embryo, erythrocyte CA activity is kept at a very low level throughout most of the incubation period $(2,7)$, with minimum activities recorded between days 8 and 12 of development, but begins to increase rapidly from day 14 onward. The control mechanisms responsible have not been identified.

We have previously shown that short- or long-term incubation of chick embryos under hypoxic conditions causes a rapid increase in CA activity of embryonic erythrocytes and a coordinate change in the erythrocyte organic phosphate pattern, i.e., a reduction of the ATP concentration and an increase in erythrocyte 2,3-diphosphoglycerate $(2,3-\mathrm{DPG})$ concentration $(1,2)$. The net result of the altered phosphate pattern is an increase of whole blood oxygen affinity (3), since 2,3-DPG is a weaker allosteric effector of avian hemoglobin than ATP
(27). During normal embryonic development these changes in erythrocyte metabolism (and oxygen affinity) occur in the prehatch period $(13,17)$ at a time when avian embryos become progressively hypoxic as well as hypercapnic, since gas transfer across the eggshell is diffusion limited in the last period of development (21). Thus, in chick embryos the $\mathrm{Po}_{2}$ of the chorioallantoic vein, which carries the oxygenated blood back to the embryo, decreases from $\sim 90$ Torr to $<50$ Torr (25), and there is a concomitant increase in $\mathrm{PCO}_{2}(25)$.

Ingermann et al. (12) could demonstrate that, after day 14 of incubation, exposure to hyperoxia suppresses the prehatch change of the organic phosphate pattern and oxygen affinity of chick embryonic erythrocytes. We likewise observed decreased erythrocyte CA activity in hyperoxic embryos (4). These data strongly suggest that a large part of the changes of embryonic erythrocyte metabolism seen during normal avian ontogeny is under control of the oxygen pressure of the embryonic blood. In the present study we have tried to identify putative hormonal signals involved in the oxygen pressure-dependent regulation of erythrocyte metabolism using changes of the erythrocyte CA activity as an indicator. We can show that after day 6 of incubation embryonic chick plasma contains a specific heat-labile low-molecular-mass factor, also found in adult chick plasma but not in mammalian plasma, that in vitro can induce a rapid de novo synthesis of CA via stimulation of transcription.

The action of this factor is completely blocked by pretreatment of erythrocytes with pertussis toxin or aluminum fluoride and reduced by treatment of erythrocytes with activators of protein kinase $\mathrm{C}$. In vivo the plasma factor does not induce de novo CA synthesis as long as blood $\mathrm{PO}_{2}$ is high, suggesting that under these conditions short-lived inhibitors are synthesized which may act on the same set of $\mathrm{G}$ proteins modulated by pertussis toxin or aluminum fluoride.

\section{METHODS}

Fertilized chicken eggs were incubated at $37.5^{\circ} \mathrm{C}$ and $60 \%$ relative humidity in a commercial incubator. To test the effects of short-term hypoxia, embryos were incubated for either 6.5 and 7.5 days in air and $12 \mathrm{~h}$ in $13.5 \% \mathrm{O}_{2}$ or for 10 days in air and $24 \mathrm{~h}$ in $13.5 \% \mathrm{O}_{2}$; for experiments under hypoxic conditions embryos were incubated for 13 days in air and subsequently in $90 \% \mathrm{O}_{2}$ for 48-96 h. Hypoxic and hyperoxic incubations were carried out in an incubator (Heräus B5060 EK2) 
equipped with an automatic control system for incubator $\mathrm{PO}_{2}$. Blood was sampled from the embryos after opening of a large extraembryonic blood vessel from which the effluent blood was aspirated with a Pasteur pipette and transferred immediately into cold buffer [50 $\mathrm{mM}$ tris(hydroxymethyl)aminomethane hydrochloride, (Tris. $\mathrm{HCl}) 120 \mathrm{mM} \mathrm{NaCl}, 4 \mathrm{mM} \mathrm{KCl}, 5 \mathrm{mM}$ glucose, and 1.5 $\mathrm{mM} \mathrm{CaCl} 2$ (pH 7.4)]. Pooled erythrocytes were washed three times with buffer before incubation.

In vitro incubations of embryonic erythrocytes in various media were carried out at $37^{\circ} \mathrm{C}$. Erythrocytes (final hematocrit $10 \%$ ) were incubated in bicarbonate-free $\mathrm{F}$ 10 medium (Boehringer Mannheim) with $20 \mathrm{mM} \mathrm{N}-2$ hydroxyethylpiperazine- $N^{\prime}$-2-ethanesulfonic acid $(\mathrm{pH}$ 7.35 ) in the absence or presence of a $10 \%$ plasma supplement and additional test substances. The samples were flushed with water vapor-saturated air and gently shaken throughout the incubation. Incubation length varied from 2 to $24 \mathrm{~h}$. At the end of the incubation erythrocytes were washed three times and stored at $-80^{\circ} \mathrm{C}$. For preparation of embryonic blood plasma whole blood was collected by venipuncture from 6 - to 18 -day-old embryos. A glass capillary connected to a syringe was inserted into a large blood vessel under microscopic control with the aid of a micromanipulator, and blood was aspirated while taking care to avoid any contamination.

Human plasma was obtained from members of the department, and adult chick plasma was obtained from White Leghorn hens. Fetal calf serum (FCS) was purchased from Boehringer Mannheim. Red cell CA activities of packed erythrocytes (expressed as $\mathrm{U} / \mathrm{g} \mathrm{Hb}$ ) were determined with the method of Maren (16) using the modification of Bruns et al. (6). Because the mean corpuscular hemoglobin concentration of embryonic chick erythrocytes changes only minimally after day 8 of incubation (25), erythrocyte CA activities are correctly assessed when related to hemoglobin concentration.

Sodium dodecyl sulfate electrophoresis of erythrocyte lysate. To test whether previously documented changes in CA activity of embryonic erythrocytes, where minimal activities were recorded between day 8 and 14 of development (2), represent true differences of the CA content of red blood cells and not activation of a preexisting enzyme pool, we have carried out sodium dodecyl sulfate (SDS) electrophoresis of erythrocyte lysates obtained from embryos between days 4 and 18 of incubation.

Packed erythrocytes were lysed by repeated freezing and thawing, and the lysate was diluted with hypotonic buffer [ $5 \mathrm{mM}$ Tris $\cdot \mathrm{HCl}, 5 \mathrm{mM} \mathrm{NaN}, 1 \mathrm{mM}$ ethylene glycol-bis ( $\beta$-aminoethyl ether)- $N, N, N^{\prime}, N^{\prime}$-tetraacetic acid, $1 \mathrm{mM}$ dithiothreitol, and $1 \mathrm{mM}$ phenylmethylsulfonyl fluoride ( $\mathrm{pH} 7.4)]$. The lysates were centrifuged at $150,000 \mathrm{~g}$ for $90 \mathrm{~min}$ at $4^{\circ} \mathrm{C}$ in a Centrikon T-2070 ultracentrifuge (Kontron Instruments, Eching, FRG) and routinely tested for contamination from DNA, RNA, and mitochondria using standard methods. The supernatant obtained after centrifugation was essentially free of contamination with RNA, DNA, and mitochondria.

The protein concentration of the lysates was determined by measuring the hemoglobin concentration, since hemoglobin accounts for $>90 \%$ of the cytoplasmic protein. SDS-polyacrylamide gel electrophoresis (PAGE) of the cytoplasmic fraction was performed according to Laemmli (14). Vertical PAGE was performed at constant current on 10 or $15 \%$ polyacrylamide gels of $1 \mathrm{~mm}$ thickness using either the minigel apparatus from Biometra (Göttingen) or the Protean II slab cell from Biorad. The SDS-PAGE standards from Biorad were diluted 1:50 in SDS sample buffer and used in 10- or 50- $\mu$ l samples. To allow internal standardization of the gels with chick CA we prepared CA from chicken erythrocytes by affinity chromatography on a Prontosil-Sephadex column (20). CA was eluted with acetazolamide (10). Gels were fixed in $12 \%$ trichloroacetic acid and stained with Coomassie Blue G-250 after the method of Neuhoff et al. (19).

Separation of primitive and definitive erythrocytes from the blood of 7- and 8-day-old chick embryos incubated for $12 \mathrm{~h}$ in $13.5 \% \mathrm{O}_{2}$ before blood sampling was carried out by density gradient centrifugation using a discontinuous gradient of Percoll (density range 1.051.11) and the gradient centrifuge Bühler ZS (Bühler, Tübingen, FRG). The percentage of primitive and definitive erythrocytes present in the collected fractions was assessed from inspection of blood smears stained with Wright-Giemsa using the histological criteria of Lucas and Jamroz (15). 2,3-DPG was determined enzymatically with a test kit (Boehringer Mannheim).

Chemicals. All reagents for electrophoresis were obtained from Biorad. Prostaglandin $\mathrm{E}_{2}$, phorbol 12myristate, 13-acetate (PMA), dexamethasone, dibutyryladenosine $3^{\prime}, 5^{\prime}$-cyclic monophosphate (cAMP), dibutyrylguanosine $3^{\prime}, 5^{\prime}$-cyclic monophosphate (cGMP), $N^{6}$ phenylisopropyladenosine (PIA), 8-phenyltheophylline, adenosine, epinephrine, norepinephrine, actinomycin D, cycloheximide, pertussis toxin, cholera toxin, arachidonic acid, carbachol, and thyroxine were obtained from Sigma. Serotonin and acetylcholine were obtained from Serva (Heidelberg, FRG), prostacyclin from Paesel (Frankfurt, FRG), dithiothreitol from Fluka, and forskolin and A23187 from Calbiochem. Calcitriol was a kind gift of Hoffmann-La Roche.

\section{RESULTS}

Effect of hyperoxia and hypoxia on erythrocyte $C A$ content during development. Figure 1 shows the results of the SDS-PAGE of erythrocyte lysates from 4- to 18day-old chick embryos. Although the Coomassie Blue stain allows only a semiquantitative estimation of protein concentration, it is obvious that the intracellular CA content is very low until day 14; thereafter it increases sharply. At the two earliest stages (days 4 and 6 ) staining of the CA band is more pronounced than at days 8-12, in agreement with the previously obtained data for CA activity (2), which were twofold higher at day 6 and sixfold higher at day 4 (compared with days 8-12).

The effect of hypoxia on erythrocyte CA content is demonstrated in Fig. 2. When 10-day-old embryos were incubated for $24 \mathrm{~h}$ in $13.5 \% \mathrm{O}_{2}$ we observed a drastic increase of the erythrocyte CA content (Fig. 2) compared with controls. The measured CA activities (means \pm SD) for the samples were $2,235 \pm 536 \mathrm{U} / \mathrm{g} \mathrm{Hb}$ for the controls and $32,509 \pm 4,961 \mathrm{U} / \mathrm{g} \mathrm{Hb}$ for the hypoxic group, i.e., a 14-fold increase within $24 \mathrm{~h}$. Note that the CA activity 


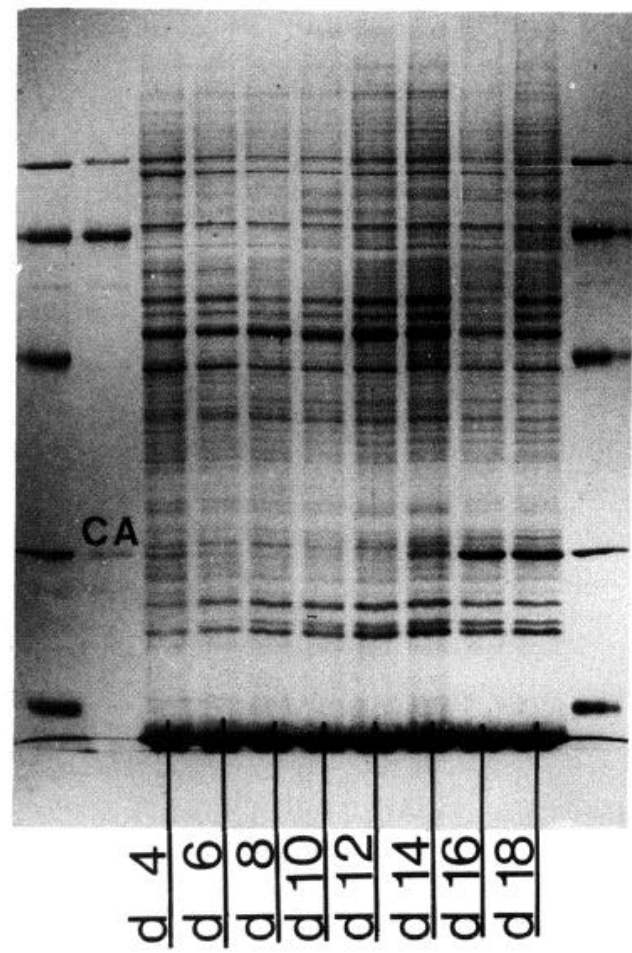

FIG. 1. SDS electrophoresis of the water-soluble cytoplasmic protein fraction of embryonic erythrocytes from 4- to 18-day-old chick embryos. CA indicates position of carbonic anhydrase on gel.

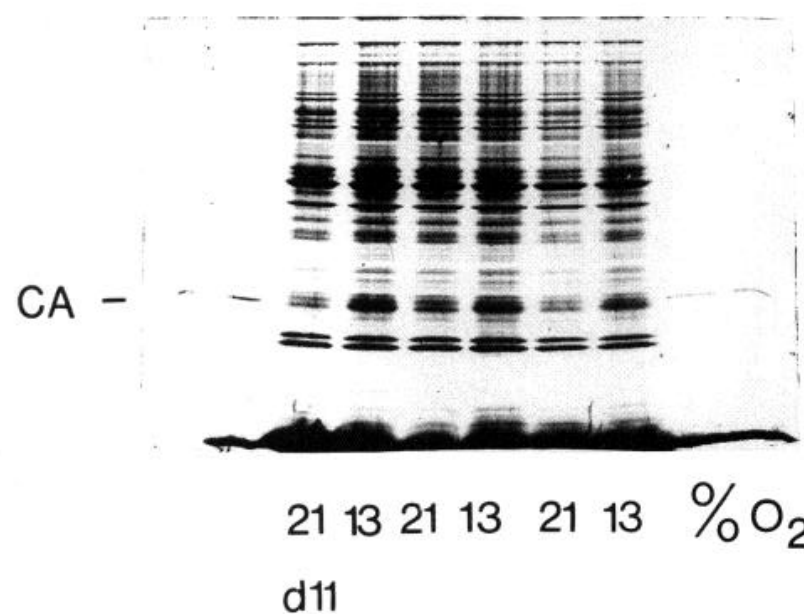

FIG. 2. SDS electrophoresis of the water-soluble cytoplasmic protein fraction of embryonic chick erythrocytes from 11-day-old embryos that had been incubated in air (control) or for 10 days in air and 1 day in $13.5 \%$ oxygen.

of adult erythrocytes is around $60,000 \mathrm{U} / \mathrm{g} \mathrm{Hb}(59,340 \pm$ $2,871 \mathrm{U} / \mathrm{g} \mathrm{Hb}, n=4$ ).

Hyperoxia had the opposite effect on CA content (Fig. 3 ). When 13-day-old embryos were incubated in $90 \% \mathrm{O}_{2}$ for 48,72 , and $96 \mathrm{~h}$, which covers the period of rapid CA increase during normoxic incubation, we found that the erythrocyte CA content was drastically reduced compared with that of the controls (Fig. 3). The individual activities of the samples tested are given in the legend to Fig. 3. These results demonstrate that the activity differences reflect changes of the CA content. Previous experiments have shown that hypoxia increases CA activity only after day 6 of development, i.e., at a time
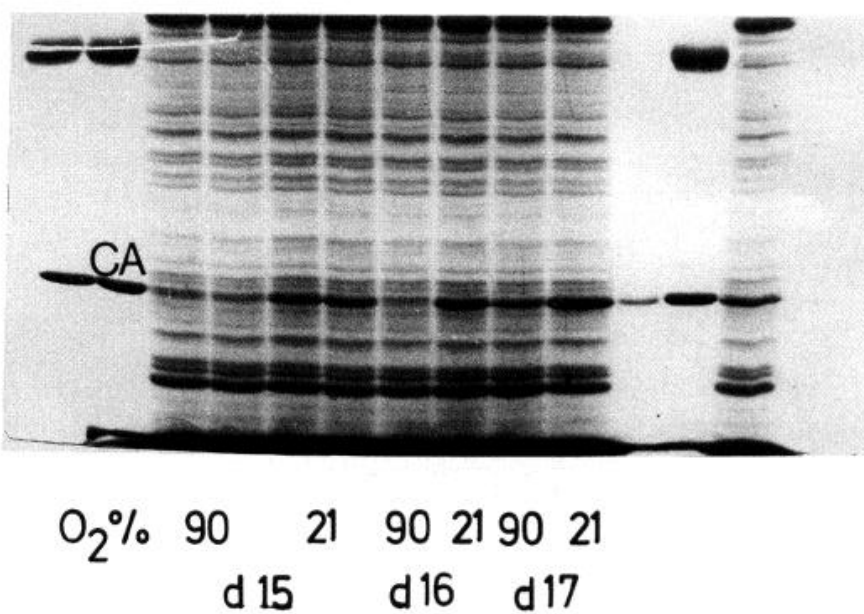

FIG. 3. SDS electrophoresis of cytoplasmic fraction of embryonic chick erythrocytes from days 15,16 , and 17 . Incubation was either in air (control) or for 13 days in air and 48,72 , and $96 \mathrm{~h}$ in $90 \%$ oxygen. CA activities of tested samples: day 15 control, $29,660 \mathrm{U} / \mathrm{g} \mathrm{Hb} ; 90 \%$ oxygen, 7,654 U/g Hb; day 16, control $35,600 \mathrm{U} / \mathrm{g} \mathrm{Hb} ; 90 \%$ oxygen, $3,685 \mathrm{U} / \mathrm{g} \mathrm{Hb}$; day 17 , control $58,374 \mathrm{U} / \mathrm{g} \mathrm{Hb} ; 90 \%$ oxygen: $11,016 \mathrm{U} / \mathrm{g}$ $\mathrm{Hb}$.

when definitive erythrocytes appear in the embryonic circulation. This suggested a different response of primitive erythrocytes (first embryonic erythrocyte population) and definitive erythrocytes to hypoxia.

Figure 4 shows the results of the 2,3-DPG and CA activity measurements of fractionated blood of 7- and 8day-old embryos exposed to hypoxia $\left(12 \mathrm{~h}\right.$ in $\left.13.5 \% \mathrm{O}_{2}\right)$ before blood sampling. It can be seen that the CA activity and 2,3-DPG concentration increase under hypoxic conditions regardless of the cell type. Linear regression analysis gave no correlation between percentage of definitive erythrocytes and CA activity of hypoxic embryos. The mean values calculated for the pooled data of either hypoxic or normoxic embryos were significantly different at the $0.1 \%$ level, with $2,744 \pm 767 \mathrm{U} / \mathrm{g} \mathrm{Hb}(n=22)$ and $6,778 \pm 1,864 \mathrm{U} / \mathrm{g} \mathrm{Hb}(n=23)$. In other words after day 6 of development primitive erythrocytes show the same response to hypoxia as definitive erythrocytes.

Influence of embryonic plasma from 6- to 18-day-old embryos on CA activity during in vitro incubation. Here, we present the results of those experiments carried out to establish whether embryonic plasma contains factors involved in the regulation of CA synthesis.

In a first set of experiments we tried to clarify if the effect of hypoxia observed in vivo can be mimicked in vitro. As test erythrocytes we selected those from 12day-old normal embryos, since they have a very low CA activity and it is comparatively easy to obtain the cells in sufficiently large quantities compared with earlier stages.

To look for potential plasma factors responsible for the CA induction, erythrocytes were incubated in media supplemented with $10 \%$ embryonic plasma from hypoxic or normoxic embryos (Table 1). After 6 h erythrocytes incubated with $10 \%$ plasma supplement obtained from 8- to 18-day-old normoxic embryos (pooled data from all incubations with plasma) had doubled their CA activity compared with that of the controls. Mean values were 

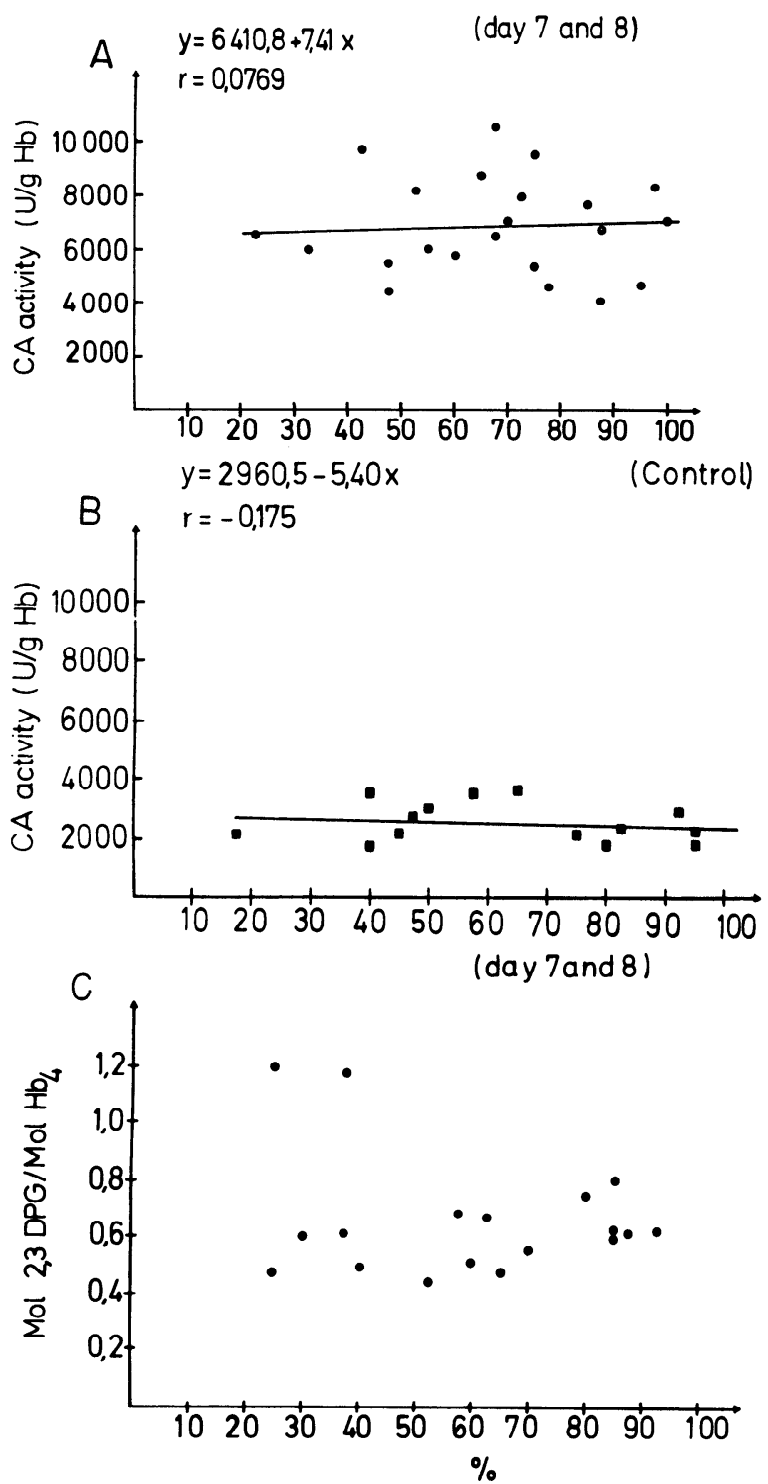

FIG. 4. $A$ and $B$ : CA activity as a function of percent of definitive erythrocytes in fractionated embryonic blood from 7- and 8-day-old chick embryos incubated in air (control) or in air and $12 \mathrm{~h}$ of $13.5 \%$ oxygen. C: 2,3-DPG concentration as a function of percent of definitive erythrocytes in fractionated blood of embryos of day 7 and 8 (shortterm hypoxia); control values for 2,3-DPG in air-incubated embryos were always $<0.1 \mathrm{~mol} 2,3-\mathrm{DPG} / \mathrm{mol} \mathrm{Hb}$.

TABLE 1. Effect of embryonic plasma on erythrocyte $C A$ activity after $6 h$ in vitro incubation

\begin{tabular}{lrl}
\hline \multicolumn{1}{c}{ Treatment } & $n$ & \multicolumn{1}{c}{$\begin{array}{c}\text { CA Activity, } \\
\text { U/g Hb }\end{array}$} \\
\hline Control (F-10 medium) & 44 & $2,751 \pm 813$ \\
+10\% Embryonic plasma (days 8-18) & 51 & $5,018 \pm 1,338^{*}$ \\
+10\% Plasma (day 11 normoxic) & 6 & $4,786 \pm 976^{*}$ \\
$+10 \%$ Plasma (day 11 hypoxic) & 5 & $4,113 \pm 655^{*}$ \\
\hline
\end{tabular}

Values are means $\pm \mathrm{SD}$. Erythrocytes from 12-day-old chick embryos were incubated for $6 \mathrm{~h}$ in $\mathrm{F}-10$ medium or in $\mathrm{F}-10+10 \%$ plasma from normoxic and hypoxic chick embryos. ${ }^{*}$ Significant difference between mean values $(2 P<0.05)$.

$2,751 \mathrm{U} / \mathrm{g} \mathrm{Hb}$ compared with $5,018 \mathrm{U} / \mathrm{g} \mathrm{Hb}(n=51 ; P<$ $0.001)$.

Preliminary measurements show an increase in 2,3DPG of 0.09 to $0.4 \mathrm{~mol} 2,3-\mathrm{DPG} / \mathrm{mol} \mathrm{Hb}$ under the same conditions (Zillner, unpublished observation). We also tested whether plasma obtained from embryos incubated under hypoxic conditions is more effective as an inducing agent (Table 1) using plasma from control embryos (day 11) and hypoxic embryos of the same age. However, we found that plasma obtained from normoxic chick embryos was as effective as plasma from hypoxic embryos (CA activity with $10 \%$ normal plasma: $4,786 \pm 976 \mathrm{U} / \mathrm{g}$ $\mathrm{Hb}, n=6 ; 10 \%$ hypoxic plasma: $4,113 \pm 655 \mathrm{U} / \mathrm{g} \mathrm{Hb}, n$ $=5$ ). Likewise plasma obtained at day 8 was as potent as plasma from day 16 or 18 ; therefore the differences of CA activity between erythrocytes from days 8 and 16 or 18 cannot be attributed to differences in the content of those plasma factors that stimulate CA production.

In contrast (Fig. 5) plasma obtained from 6-day-old embryos failed to stimulate CA production of erythrocytes (12-day embryos), and it can also be seen that primitive erythrocytes from day 6 did not increase their CA activity when they were incubated with plasma from day 6 or 18 . Both the cellular receptor for the CAinducing plasma factor as the well the factor itself are not present or active up to day 6 of development, thus explaining why hypoxic incubation is without effect on $\mathrm{CA}$ activity at this stage of development. Figure 5 also contains the results of those experiments where we tested the effectiveness of plasma from other sources, notably adult chick plasma, human adult plasma, and FCS. Although all preparations from chick were equally effective, mammalian plasma failed to stimulate CA synthesis (Fig. $5)$.

Changes of $C A$ activity during $24 h$ in vitro incubation. The time course of the CA activity change during in vitro incubation is shown in Fig. 6, where cells from day 12 were incubated up to $24 \mathrm{~h}$ either in control medium or with medium F-10. In the presence of plasma a continuous increase in CA activity (after an initial lag phase of

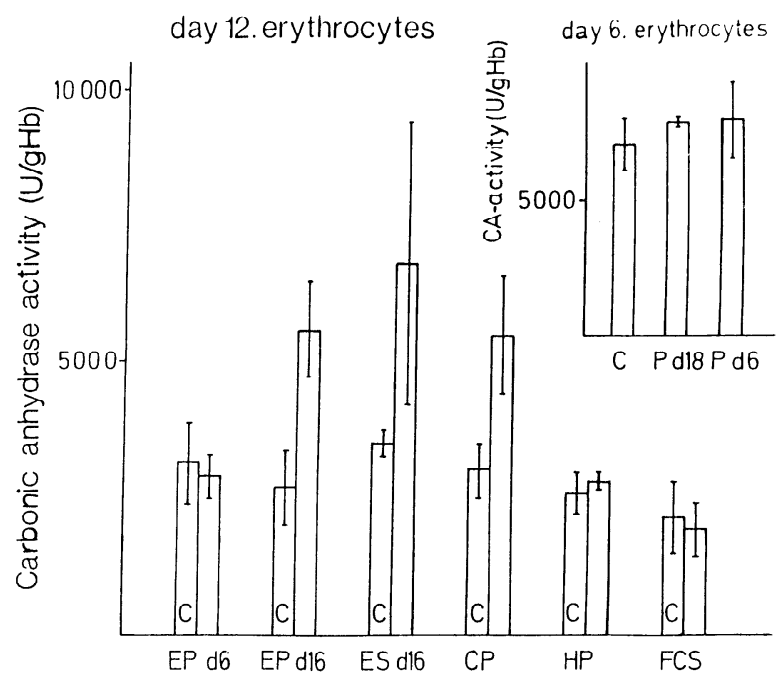

FIG. 5. CA activity (means \pm SD from at least 3 experiments) of embryonic chick red cells from 12-day-old embryos after $6 \mathrm{~h}$ incubation in the following media: medium F-10 supplemented with $10 \%$ of embryonic plasma from day 6 ( $\mathrm{EP} \mathrm{d} 6)$; embryonic plasma from day 16 (EP d16); embryonic serum from day 16 (ES d16); adult chick plasma (CP); human plasma (HP); fetal calf serum (FCS). Inset: CA activity of red cells from 6-day-old embryo after incubation $(6 \mathrm{~h})$ with medium $\mathrm{F}-10$ and $10 \%$ plasma from day $6(\mathrm{P} \mathrm{d} 6)$ and day $18(\mathrm{P} \mathrm{d} 18)$, respectively. Controls were incubated with medium $\mathrm{F}-10$ 


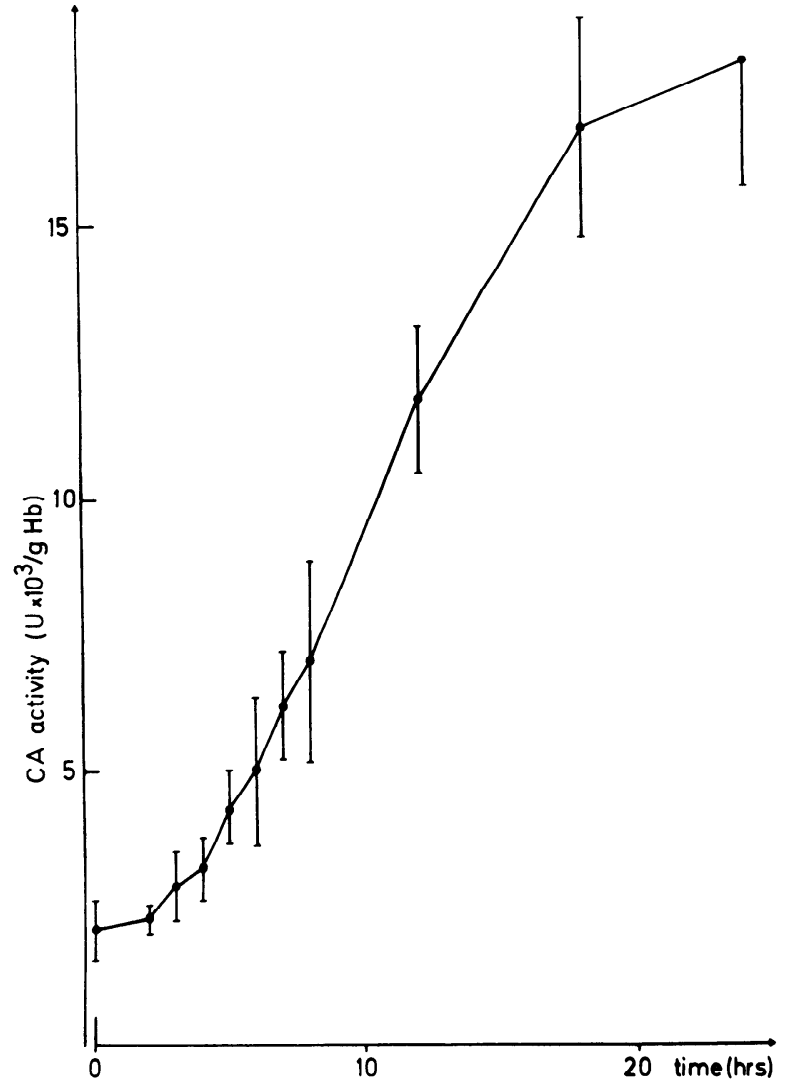

FIG. 6. Change in CA activity of erythrocytes harvested from 12day-old embryo during $24 \mathrm{~h}$ incubation with medium F-10 and $10 \%$ embryonic plasma from 14- to 16-day-old embryos. Controls were incubated in medium F-10 and had a CA activity of $2,125 \pm 550 \mathrm{U} / \mathrm{g}$ $\mathrm{Hb}$ after $24 \mathrm{~h}$. Data presented are from at least 3 experiments at each point.

$\sim 2 \mathrm{~h}$ ), which levels off between 20 and $24 \mathrm{~h}$, is observed. The total activity increase during $24 \mathrm{~h}$ is nearly 10 -fold, from $2,125 \mathrm{U} / \mathrm{g} \mathrm{Hb}$ in the controls to $18,015 \mathrm{U} / \mathrm{g} \mathrm{Hb}$ in cells incubated with $10 \%$ plasma.

Effect of actinomycin D, cycloheximide, and heat inactivation of plasma. To establish at which level the plasma factor stimulates CA synthesis, incubations were carried out in the presence of actinomycin D and cycloheximide (Table 2). Inasmuch as both substances were inhibitory, one can conclude that stimulation of CA synthesis requires activation of transcription. Furthermore, the plasma effect on CA production was abolished when plasma was heated for $10 \mathrm{~min}$ at $95^{\circ} \mathrm{C}$, indicating that the factor is heat labile. On the other hand trypsinization or acid treatment had no effect. To determine the size of the factor, plasma was fractionated using a series of ultrafilters (Amicon) with nominal molecular mass cutoff varying from 30,000 (PM30) to 2,000 Da (YM2). Stimulation of CA activity was obtained with all ultrafiltrates regardless of the filter used. When erythrocytes were incubated for $6 \mathrm{~h}$ with ultrafiltrate that had passed through an Amicon YM2 filter (nominal cutoff 2,000 $\mathrm{Da}$ ), the CA activity increased to $5,800 \pm 1,025 \mathrm{U} / \mathrm{g} \mathrm{Hb}$, $n=4$ compared with $3,132 \pm 804 \mathrm{U} / \mathrm{g} \mathrm{Hb}$ in the controls. Therefore the molecular mass of the factor must be $<2,000 \mathrm{Da}$.

Influence of hormones and intracellular messengers. To
TABLE 2. Inhibition of plasma-induced CA activity increase during 6 h incubation under various conditions

\begin{tabular}{lrll}
\hline \hline \multirow{2}{*}{ Incubation } & & \multicolumn{2}{c}{ CA Activity, U/g Hb } \\
\cline { 3 - 4 } & $n$ & $\begin{array}{c}\text { Control (F-10 } \\
\text { medium + 10\% } \\
\text { plasma) }\end{array}$ & With supplement \\
\hline Actinomycin D (35 $\mu \mathrm{M})$ & 5 & $5,244 \pm 937$ & $2,617 \pm 502^{*}$ \\
Cycloheximide (50 $\mu \mathrm{M})$ & 5 & & $2,617 \pm 132^{*}$ \\
Heat-inactivated plasma & 10 & $4,465 \pm 1,315$ & $2,402 \pm 323^{*}$ \\
Acidified plasma & 3 & $5,471 \pm 1,796$ & $5,410 \pm 1,679$ \\
Trypsinized plasma & 3 & $5,471 \pm 1,796$ & $5,330 \pm 1,000$ \\
\hline
\end{tabular}

Values are means \pm SD. Erythrocytes from 12-day-old embryos were incubated in vitro for $6 \mathrm{~h}$ at $37^{\circ} \mathrm{C}$ with $\mathrm{F}-10$ medium $+10 \%$ embryonic plasma (control) or with $10 \%$ plasma $+35 \mu \mathrm{M}$ actinomycin D or $10 \%$ plasma $+50 \mu \mathrm{M}$ cycloheximide. In the other experiments normal plasma was substituted by $10 \%$ modified plasma + heat treatment $(10$ min at $95^{\circ} \mathrm{C}$ ), acidified plasma (60 min, $\mathrm{pH} 3$, with subsequent neutralization), or trypsin $(0.1 \% 60 \mathrm{~min}$ at room temperature $) .{ }^{*}$ Significant difference between mean values $(2 P<0.05)$.

identify possible transduction pathways for the plasma factor we screened a variety of hormones, second messengers, and $\mathrm{G}$ protein modulators. The combined results are shown in Tables 3 and 4, respectively. Calicitriol, thyroxine, and epinephrine, which have been shown to alter CA expression in various tissues $(18,24)$, were without effect; likewise direct stimulation of adenylate cyclase with forskolin or incubation with cAMP did not alter the response of the cells to plasma nor did it induce de novo CA formation. Similarly negative results were obtained with prostaglandins, cGMP, norepinephrine, dexamethasone, and ATP. Carbachol and serotonin were also ineffective. However, we found that we could completely suppress the plasma effect when erythrocytes were treated with pertussis toxin or aluminum fluoride (Table 3). We also observed a significant reduction of the response when cells were incubated either in the presence of the phorbol ester PMA, which activates protein kinase $\mathrm{C}$, or with the calcium ionophore $\mathrm{A} 23187$ (Table 3). On the other hand an increase of the CA activity in the absence of plasma was only observed with the $\mathrm{A}_{1}$-receptor agonist PIA used in high concentration (i.e., $20 \mu \mathrm{M}$ ); lower concentrations were without effect (Table 4). Note also that the effect of PIA is not additive to that of plasma.

The data presented in Table 5 compare the effects of PIA, adenosine, and plasma under various conditions. In particular it was found that the physiological agonist adenosine at $20 \mu \mathrm{M}$ failed to increase CA activity (Table 5). Treatment of plasma with adenosine deaminase $(0.5$ $\mathrm{U} / \mathrm{ml}$ ) did not alter the potency with regard to CA stimulation, nor did incubation in the presence of the $\mathrm{A}_{1}$-receptor antagonist 8-phenyltheophylline $(20 \mu \mathrm{M})$ suppress the plasma effect. 8-Phenyltheophylline was, however, effective in suppressing the effect of PIA (Table 5). Interestingly the effect of PIA was also abolished in the presence of pertussis toxin, aluminum fluoride, actinomycin D, and PMA. Taken together these results strongly argue against adenosine as the mediator of the plasma effect; they suggest, however, that the receptor for the plasma factor and the $A_{1}$ receptor activated by 
TABLE 3. Influence of second messengers and $G$ protein modifiers on $C A$ activity during 6 in vivo incubation

\begin{tabular}{|c|c|c|c|c|}
\hline \multirow{2}{*}{ Tested Substance } & \multicolumn{4}{|c|}{ CA Activity, U/g Hb } \\
\hline & $\mathrm{I}$ & II & III & IV \\
\hline Forskolin $(1 \mu \mathrm{M})$ & $2,354 \pm 372$ & $2,434 \pm 705$ & $6,173 \pm 1,402^{*}$ & $6,100 \pm 1,221^{*}$ \\
\hline$n$ & 5 & 6 & 3 & 4 \\
\hline $\begin{array}{l}\text { Dibutyryl-cAMP (0.1 mM) } \\
n\end{array}$ & $\begin{array}{c}2,370 \pm 428 \\
5\end{array}$ & $2,459 \pm \frac{5}{6} 596$ & $\underset{3}{6,263 \pm 1,026^{*}}$ & $\frac{5,693 \pm 1,651^{*}}{3}$ \\
\hline Dibutyryl-cGMP (10 $\mu \mathrm{M})$ & $3,112 \pm 992$ & $2,996 \pm 716$ & $4,506 \pm 1,311^{*}$ & $4,724 \pm 1,622^{*}$ \\
\hline$n$ & 5 & 5 & 3 & 3 \\
\hline PMA $(0.1 \mu \mathrm{M})$ & $3,008 \pm 975$ & $3,566 \pm 825$ & $4,642 \pm 956^{*}$ & $3,321 \pm 123$ \\
\hline$n$ & 6 & 5 & 3 & 3 \\
\hline $\mathrm{A} 23187(0.1 \mu \mathrm{M})$ & $3,008 \pm 975$ & $2,777 \pm 1,115$ & $4,642 \pm 956^{*}$ & $3,539 \pm 627$ \\
\hline$n$ & 6 & 3 & 3 & 3 \\
\hline $\mathrm{NaF}(10 \mathrm{mM})+\mathrm{AlCl}_{3}(10 \mu \mathrm{M})$ & $2,873 \pm 373$ & $2,744 \pm 584$ & $4,925 \pm 575^{*}$ & $2,471 \pm 555$ \\
\hline$n$ & 6 & 3 & 8 & 10 \\
\hline Cholera toxin $(0.1 \mu \mathrm{M})$ & $3,043 \pm 970$ & 2,471 & $6,027 \pm 1,194^{*}$ & $5,676 \pm 1,023^{*}$ \\
\hline$n$ & 6 & & 5 & 7 \\
\hline Pertussis toxin $(0.1 \mu \mathrm{M})$ & $3,043 \pm 970$ & & $6,027 \pm 1,194^{*}$ & $3,290 \pm 1,297$ \\
\hline$n$ & 6 & & 5 & 7 \\
\hline
\end{tabular}

Values are means \pm SD. Effect of various second messengers and G protein modulators on CA activity. Erythrocytes from 12-day-old chick embryos were incubated for $6 \mathrm{~h}$ at $37^{\circ} \mathrm{C}$ in F-10 medium with various supplements. I, control incubation (F-10 medium); II, F-10 medium + test substance; III, F-10 medium $+10 \%$ embryonic plasma; IV, F-10 medium $+10 \%$ embryonic plasma + test substance. ${ }^{*}$ Significant difference between mean values $(2 P<0.05)$.

TABLE 4. Influence of various hormones and precursors on CA activity during 6 in vitro incubation

\begin{tabular}{|c|c|c|c|c|}
\hline \multirow{2}{*}{ Hormone or Precursor } & \multicolumn{4}{|c|}{ CA Activity, U/g Hb } \\
\hline & I & II & III & IV \\
\hline$\underset{n}{\operatorname{Arachidonic} \text { acid }(1 \mu \mathrm{M})}$ & $\frac{2,504 \pm 476}{5}$ & $\begin{array}{c}2,375 \pm 193 \\
3\end{array}$ & $\begin{array}{c}4,867 \pm 858^{*} \\
8\end{array}$ & $\frac{5,357 \pm 1,656^{*}}{6}$ \\
\hline$\underset{n}{\text { Prostaglandin } \mathrm{E}_{2}(1 \mu \mathrm{M})}$ & $\begin{array}{c}2,296 \pm 332 \\
6\end{array}$ & $\frac{2,356 \pm 502}{6}$ & $6,937 \pm 463^{*}$ & $\begin{array}{c}7,084 \pm 1,236^{*} \\
3\end{array}$ \\
\hline Prostacyclin $(1 \mu \mathrm{M})$ & $2,641 \pm 758$ & $2,535 \pm 753$ & $4,862 \pm 141^{*}$ & $4,761 \pm 1,210^{*}$ \\
\hline$n$ & 4 & 3 & 3 & 5 \\
\hline ATP $(10 \mu \mathrm{M})$ & $3,891 \pm 530$ & $3,651 \pm 521$ & $6,950 \pm 848^{*}$ & $6,375 \pm 1,732^{*}$ \\
\hline$n$ & 3 & 3 & 3 & 3 \\
\hline $\begin{array}{l}\text { Epinephrine }(10 \mu \mathrm{M}) \\
n\end{array}$ & $\begin{array}{c}2,625 \pm 714 \\
5\end{array}$ & $\begin{array}{c}2,880 \pm 764 \\
5\end{array}$ & $\begin{array}{c}4,766 \pm 647^{*} \\
4\end{array}$ & $\begin{array}{c}5,249 \pm 1,245^{*} \\
3\end{array}$ \\
\hline Norepinephrine $(10 \mu \mathrm{M})$ & $2,319 \pm 530$ & $2,447 \pm 422$ & $4,566 \pm 899^{*}$ & $4,348 \pm 1,095^{*}$ \\
\hline$n$ & 3 & 3 & 3 & 3 \\
\hline Calcitriol $(0.1 \mu \mathrm{mol})$ & $2,185 \pm 427$ & $1,930 \pm 520$ & $4,660 \pm 850^{*}$ & $4,547 \pm 1,095^{*}$ \\
\hline$n$ & 3 & 3 & 5 & 4 \\
\hline Thyroxine (10-100 nM) & $2,078 \pm 856$ & $2,498 \pm 626$ & $4,359 \pm 881^{*}$ & $4,426 \pm 710^{*}$ \\
\hline$n$ & 4 & 4 & 3 & 6 \\
\hline Dexamethasone $(0.01-1 \mu \mathrm{M})$ & $3,046 \pm 473$ & $2,862 \pm 591$ & $5,900 \pm 1,033^{*}$ & $5,568 \pm 1,496^{*}$ \\
\hline$n$ & 8 & 7 & 9 & 5 \\
\hline PIA $(20 \mu \mathrm{M})$ & $3,141 \pm 663$ & $4,940 \pm 982^{*}$ & $5,488 \pm 1,082^{*}$ & $4,890 \pm 750^{*}$ \\
\hline$n$ & 6 & 7 & 4 & 3 \\
\hline Serotonin $(0.1 \mu \mathrm{M})$ & $3,626 \pm 663$ & $3,618 \pm 715$ & $6,226 \pm 1,364^{*}$ & $6,329 \pm 820^{*}$ \\
\hline$n$ & 3 & 3 & 3 & 3 \\
\hline Carbachol $(10-100 \mu \mathrm{M})$ & $3,251 \pm 293$ & $3,400 \pm 483$ & $6,490 \pm 470^{*}$ & $6,459 \pm 1,098^{*}$ \\
\hline$n$ & 3 & 3 & 3 & 4 \\
\hline
\end{tabular}

Values are means \pm SD. Influence of various hormones on CA activity. Erythrocytes were incubated for $6 \mathrm{~h}$ in $\mathrm{F}-10 \mathrm{medium}$ with various supplements. I, control incubation (F-10 medium); II, F-10 medium + test substance; III, F-10 medium + $10 \%$ embryonic plasma; IV, F-10 medium $+10 \%$ embryonic plasma + test substance. ${ }^{*}$ Significant difference of mean values $(2 P<0.05)$.

PIA may converge on the same intramembraneous transduction structure.

\section{DISCUSSION}

The results of the present study support the concept that blood oxygen pressure plays an important role in the regulation of chick embryonic erythrocyte metabolism during normal embryonic development. This is not the result of a direct response of erythrocytes to changes in $\mathrm{PO}_{2}$ but apparently relies on the mediation via circu- lating plasma factors. The experimental findings suggest the presence of a control system of stimulatory and inhibitory plasma factors and show that erythrocyte CA expression and, by inference, also 2,3-DPG synthesis are for a large part of development under negative control.

Broadly speaking, two phases of development can be identified. The first phase, terminating at day 6 , is characterized by a low level of constitutive expression of CA, which is not susceptible to external modulation via ambient $\mathrm{PO}_{2}$ and circulating plasma factors. In vitro results show that, at this stage, the plasma does not contain the 
TABLE 5. Effect of adenosine and PIA on CA activity

\begin{tabular}{lll}
\hline \hline \multicolumn{1}{c}{ Condition } & $n$ & $\begin{array}{c}\text { CA Activity, } \\
\mathrm{U} / \mathrm{g}\end{array}$ \\
\hline Control & & $2,780 \pm 543$ \\
PIA $(20 \mu \mathrm{M})$ & 5 & $4,945 \pm 1,152^{*}$ \\
PIA + PMA $(0.1 \mu \mathrm{mol})$ & 3 & $2,879 \pm 533$ \\
PIA + PMA + A23187 $(0.1 \mu \mathrm{mol})$ & 3 & $2,414 \pm 202$ \\
PIA $(20 \mu \mathrm{M})+8-\mathrm{PT}(20 \mu \mathrm{M})$ & 3 & $2,796 \pm 416$ \\
PIA + actinomycin D $(35 \mu \mathrm{M})$ & 3 & $2,407 \pm 714$ \\
PIA + pertussis toxin $(0.1 \mu \mathrm{M})$ & 3 & $2,357 \pm 757$ \\
PIA + NaF $(10 \mathrm{mM})+\mathrm{AlCl} l_{3}(10 \mu \mathrm{M})$ & 3 & $2,933 \pm 582$ \\
Adenosine $(20 \mu \mathrm{M})$ & 4 & $2,574 \pm 239$ \\
$10 \%$ Plasma & 4 & $7,288 \pm 1,442^{*}$ \\
$10 \%$ Plasma + ADA $(0.5 \mathrm{U} / \mathrm{ml})$ & 3 & $7,952 \pm 672^{*}$ \\
$10 \%$ Plasma $+8-\mathrm{PT}(20 \mu \mathrm{M})$ & 3 & $7,249 \pm 233^{*}$
\end{tabular}

Values are means \pm SD. CA activities of erythrocytes from 12-dayold embryos incubated for $8 \mathrm{~h}$ in F-10 medium with $N^{6}$-phenylisopropyladenosine (PIA), adenosine, or plasma under various conditions. Controls: incubation in F-10 medium; ADA, adenosine deaminase; 8PT, 8-phenyltheophylline. * Significant difference from control $(2 P<$ $0.05)$.

substances responsible for stimulation of CA synthesis; in addition the primitive erythrocytes seem to lack the specific cellular receptors for binding of these factors. The second phase extends from day 7 to about day 14 and is also characterized by a very low expression of CA, which in this phase, however, is the result of active repression. Under normoxic conditions the CA activity increases from day 14 onward; this is correlated with the progressive fall in $\mathrm{Po}_{2}$ in the chorioallantoic capillary bed and the chorioallantoic vein. When blood $\mathrm{Po}_{2}$ is artificially increased during this phase by raising the ambient $\mathrm{PO}_{2}$, the de novo synthesis of $\mathrm{CA}$ is suppressed. Conversely, hypoxia elicits an increase in CA synthesis at any time after day 6 , and both definitive and old primitive erythrocytes respond.

We now turn to the evidence for the presence of plasma factors that stimulate or suppress CA synthesis. Evidence for the presence of a plasma factor that activates $\mathrm{CA}$ synthesis and causes changes in organic phosphate pattern rests on several results. First, during in vitro incubation embryonic erythrocytes from day 12 , which have a very low CA activity, begin to synthesize CA (and 2,3-DPG) when they are reincubated with their own plasma in concentrations as low as $10 \%$. This is not observed with serum-free medium. Second, the effect is lost with heat treatment of chick plasma and can be abolished by incubation with pertussis toxin, aluminum fluoride, cycloheximide, and actinomycin D. Third, ultrafiltration experiments indicate that the factor has a molecular mass of $<2,000 \mathrm{Da}$. Fourth, the synthesis of the factor changes during ontogeny. It is not present in plasma of embryos that are $<7$ days. Incubation of erythrocytes from day 12 with plasma from day 6 does not elevate CA activity. Finally, the factor seems to be specific for chick or avian plasma, since incubation with FCS or human plasma brought negative results.

Evidence for the presence of an inhibitory factor is only indirect. Thus, although the plasma factor that stimulates CA synthesis is present from day 7 onward, the CA activity in vivo is low until day 14. This result suggests that, during this time, an inhibitor which antagonizes the actions of the stimulatory plasma factor is present in the embryonic plasma. Furthermore, the inhibitor must be short lived. Indirect evidence for this concept is provided by the in vitro results. The fact that erythrocytes, which in vivo have a low activity of $\mathrm{CA}$, begin to synthesize CA when they are reincubated with their own plasma in air can only be explained by the absence of the inhibitor.

We suggest that the inhibitor molecule is synthesized in dependence of ambient $\mathrm{PO}_{2}$ and that it is only synthesized above a certain threshold value for the blood $\mathrm{Po}_{2}$. Whenever $\mathrm{Po}_{2}$ falls below the threshold level, the inhibitor is no longer synthesized in sufficient quantity and the stimulatory plasma factors activate CA synthesis as well as the parallel changes of phosphate metabolism.

Measurements of $\mathrm{Po}_{2}$ of the chorioallantoic vein show that, during normal ontogeny, it decreases from $\sim 70$ to $<50$ Torr between days 14 and 17 (25). Inasmuch as coordinate changes in CA activity and phosphate metabolism are observed from day 14 onward, it is tempting to speculate that the blood $\mathrm{Po}_{2}$ at day 14 corresponds to the threshold level below which the synthesis of the inhibitor starts to decrease. This reasoning is also supported by the finding that after day 14 experimental hyperoxia is able to suppress the change of CA and organic phosphate synthesis.

The physiological benefit of this $\mathrm{Po}_{2}$-dependent regulatory system for the avian embryo is readily apparent. It induces a rapid adaptive change of the oxygen transport system to hypoxic stress at any time after day 6 of development and therefore increases the chance for embryonic survival under these conditions.

That erythrocyte CA synthesis is regulated by a $\mathrm{Po}_{2}-$ dependent control system makes sense, since in the prehatch period hypoxia and hypercapnia develop at the same time. The increase of the CA activity helps to curb the noxious effects of respiratory acidosis on oxygen transport. The physiological importance of these adaptations of embryonic erythrocytes to the hypoxic conditions of the prehatch period is indicated by the fact that this is the period of the highest mortality during embryonic development (22), where hypoxia is certainly a major contributing factor. In contrast to the hypercapnia and hyperoxia present in late development the early to midterm embryo has a high $\mathrm{Po}_{2}$ in the chorioallantoic vein (90 Torr) and a low $\mathrm{PCO}_{2}$, which is 10 Torr at day 10 and $\sim 17$ Torr at day 13 (25). Furthermore, capillary contact time for passage of the chorioallantois is long, i.e., $\sim 1 \mathrm{~s}$ at day 10 , but decreases to $0.55 \mathrm{~s}$ at day 14 (25). Thus the absence of diffusion limitation for carbon dioxide transfer during early embryonic development and the initially long capillary contact times allow efficient carbon dioxide release from the embryo even with the low erythrocyte CA activities found before day 14 .

We now turn to an analysis of the cellular mechanism that are involved in the regulation of CA synthesis. The time course of the CA activity change during in vitro incubation with its prominent lag phase and the inhibitory effect of actinomycin $\mathrm{D}$ indicate that increased CA synthesis is due to transcriptional activation. Furthermore, the finding that the CA activity change during in vitro incubation with $10 \%$ plasma is nearly as large as that observed during in vivo incubation under hypoxic 
conditions demonstrates a remarkable in vitro stability of the stimulatory plasma activity and suggests that it is present in sufficiently high concentration to saturate the receptor binding sites, since incubation in 40 or $60 \%$ plasma did not significantly increase CA synthesis.

Our in vitro results demonstrate that of all tested compounds only the adenosine analogue PIA was able to stimulate CA synthesis and that aluminum fluoride, pertussis toxin, actinomycin D, and PMA block both the effects of PIA and plasma. Therefore one question is whether adenosine is the stimulatory plasma factor. Several results argue against adenosine. In the first place adenosine even at $20 \mu \mathrm{mol}$ was not able to stimulate CA synthesis, and PIA did so only when used at very high concentration $(>10 \mu \mathrm{mol})$. Treatment of plasma with adenosine aminase did not abolish its ability to stimulate CA synthesis, nor was it inhibited by the $A_{1}$ receptor antagonist 8-phenyltheophylline, which completely blocked the effect of PIA. The results are best compatible with the hypothesis that the $A_{1}$ receptor activated by PIA uses some of the transduction structures involved in the mediation of the plasma effect. Support for this hypothesis comes from the finding that the effect of PIA is not additive to that of plasma and also inhibitable by pertussis toxin and aluminum fluoride. There is no evidence from our results that a decrease or increase of the intracellular cAMP or cGMP concentration serves as intracellular signal, ruling out modulation of adenylate cyclase or guanylate cyclase activity as principal events in the transduction process. However, it has recently been shown that adult turkey erythrocytes possess a phospholipase $C$ (PLC) which is activated by $G_{o}$ proteins and that treatment of these cells with aluminum fluoride, an unspecific activator of $G$ proteins (23), causes increased phosphoinositide hydrolysis (11) with subsequent production of inositol 1,4,5-trisphosphate and diacylglycerol.

In other systems it has been demonstrated that PLC can be regulated by $\mathrm{G}_{\mathrm{i}}$ proteins that are pertussis toxin sensitive $(5,8,26)$. Thus activation of $A_{1}$ receptors with PIA causes a decrease of PLC activity in $\mathrm{GH}_{3}$ cells, which is abolished after treatment with pertussis toxin (8). These data suggest the following tentative explanation for our in vitro findings. Binding of the CA synthesis stimulating plasma factor to its receptor causes inhibition of PLC via a $G_{i}$ protein that can also be coupled to $A_{1}$ receptors activated by PIA. The inactivation of $G_{i}$ by pertussis toxin and concomitant activation of PLC causes an increase of inositol 1,4,5-trisphosphate and diacylglycerol, with subsequent activation of protein kinase $\mathrm{C}$ (PKC). These events inhibit de novo synthesis of CA. That PKC is involved in the signaling process for the action of inhibitors of CA synthesis is indicated by the inhibitory effects of PMA and A23187 on CA production in the presence of PIA or embryonic plasma. The putative inhibitor molecule could thus act at least on two levels: it could affect the $G$ proteins coupled to PLC or act directly on PKC. The validity of this concept has to be tested by further experiments. Finally the observation that the plasma factor is present in adult chick plasma (but not in mammalian plasma) raises the question of its function in adult birds after the conclusion of embryonic development.

Although the experiments described by us were carried out only with one species of avian embryo, we believe that the results can be transferred to other avian embryos as well in view of the remarkable homogeneity of the ontogenetic changes of erythrocyte populations and erythrocyte metabolism. In particular, the prehatch changes of the phosphate pattern and blood oxygen affinity have been demonstrated in every species investigated so far (13); comparative data for CA are lacking. Finally, the question of which structures may be responsible for the production of both the inhibitor and the activator during embryonic development must be asked. In this regard a strong case can be made for the chorioallantois; it resumes its function as the principal embryonic gas exchanger by about day 7 of development (9) at the same time when $\mathrm{PO}_{2}$-dependent regulation of erythrocyte $\mathrm{CA}$ activity and phosphate pattern is first apparent. Moreover, changes of ambient $\mathrm{Po}_{2}$ directly alter the endcapillary $\mathrm{PO}_{2}$ as well as the $\mathrm{Po}_{2}$ of the chorioallantoic vein, whereas intraembryonic structures are much less affected because of the presence of a central circulatory shunt. The evolution of a flexible system of $\mathrm{PO}_{2}$-dependent control of erythrocyte metabolism and function in avian embryos may have contributed to the ability of birds to breed at higher altitudes than all other terrestrial vertebrates.

We are grateful for the excellent technical assistance of Luise Ranch and Ernestine Jartler.

Address reprint requests to $R$. Baumann.

Received 17 October 1990; accepted in final form 21 May 1991.

\section{REFERENCES}

1. Baumann, R., J. Fischer, AND M. EnGElke. Functional properties of primitive and definitive red cells from chick embryo: oxygenbinding characteristics, $\mathrm{pH}$ and membrane potential, and response to hypoxia. J. Exp. Zool. Suppl. 1: 227-238, 1987.

2. Baumann, R., E. A. Haller, U. Schöning, and M. Weber. Hypoxic incubation leads to concerted changes of carbonic anhydrase activity and 2,3DPG concentration of chick embryo red cells. Dev. Biol. 116: 548-551, 1986.

3. Baumann, R., S. Padeken, E. A. Haller, and T. Brilmayer. Effects of hypoxia on oxygen affinity, hemoglobin pattern, and blood volume of early chicken embryos. Am. J. Physiol. 244 (Regulatory Integrative Comp. Physiol. 13): R733-R741, 1983.

4. Baumann, R., P. Zillner, ANd D. Million-Ferreira da Silva. Oxygen pressure regulates the carbonic anhydrase activity in definitive and mature primitive red cells from chick embryo (Abstract). Pfluegers Arch. 413, Suppl. 1: R12, 1989.

5. Bizzari, C., M. di Girolamo, M. C. D’Orazio, and D. Corda. Evidence that a guanine nucleotide binding protein linked to a muscarinic receptor inhibits directly phospholipase C. Proc. Natl. Acad. Sci. USA 87: 4889-4893, 1990.

6. Bruns, W., R. Dermietzel, and G. Gros. Carbonic anhydrase in the sarcoplasmatic reticulum of rabbit skeletal muscle. J. Physiol. Lond. 371: 351-364, 1986.

7. Clark, A. M. Carbonic anhydrase activity during embryonic development. J. Exp. Biol. 28: 332-343, 1951.

8. Delahunty, T. M., M. J. Cronin, and J. Linden. Regulation of $\mathrm{GH}_{3}$-cell function via adenosine $\mathrm{A}_{1}$ receptors. Biochem. J. 255: 69$77,1988$.

9. DUNCKER, H. R. Development of the avian respiratory and circulatory systems. In: Respiratory Function in Birds, Adult and Embryonic, edited by J. Piiper. Berlin: Springer-Verlag, 1978, p. 260273.

10. Falkbring, S. O., P. O. Göthe, P. O. Nyman, L. Sundberg, and 
J. PoRATh. Affinity chromatography of carbonic anhydrase. FEBS Lett. 24: 229-232, 1972.

11. Harden, T. K., P. T. Hawkins, I. Stephens, J. I. Boyfr, and C. P. DownEs. Phosphonositide hydrolysis by guanosine $5^{\prime}$-[thio] triphosphate-activated phospholipase $\mathrm{C}$ of turkey erythrocyte membranes. Biochem. J. 252: 583-593, 1988.

12. Ingermann, R. L., M. K. Stock, J. Metcalfe, and T. B. Shi. Effect of ambient oxygen on organic phosphate concentration in erythrocytes of the chick embryo. Respir. Physiol. 51: 141-152, 1983.

13. Isaacks, R. E., D. R. Harkness, R. N. Sampsell, J. L. Adler, C. Y. KIM, AND P. H. Goldman. Studies on avian erythrocyte metabolism. IV. Relationship between major phosphorylated metabolic intermediates and oxygen affinity of whole blood in adults and embryos of several galliforms. Comp. Biochem. Physiol. A Comp. Physiol. 55: 29-33, 1976.

14. LAEMmLI, U. K. Cleavage of structural proteins during the assembly of the head of bacteriophage T4. Nature Lond. 277: 680-688, 1970.

15. LuCas, A., AND C. Jamroz. Atlas of Avian Hematology. Washington, DC: US Dept. of Agriculture, 1961. (Agric. monogr. 25)

16. Maren, T. H. A simplified micromethod for the determination of carbonic anhydrase and its inhibitors. J. Pharmacol. Exp. Ther. 130: 26-29, 1960.

17. Misson, B. H., And B. M. Freeman. Organic phosphates and oxygen affinity of chick blood before and after hatching. Respir. Physiol. 14: 343-352, 1972.

18. Narbaitz, R., S. KaCEW, AND L. Sitwell. Carbonic anhydrase activity in the chick embryo chorioallantois: regional distribution and vitamin D regulation. J. Embryol. Exp. Morphol. 65: 127-137, 1978.

19. Nfuhoff, V., N. Arold, D. Taube, and W. Ehrhardt. Improved staining of proteins in polyacrylamide gels including isoelectric focusing gels with clear background at nanogram sensitivity using Coomassie Brilliant Blue G 250/R 250. Electrophoresis 9: 255-262, 1988.

20. Osborne, W. R. A., AND R. E. Tashian. An improved method for the purification of carbonic anhydrase isozymes by affinity chromatography. Anal. Biochem. 64: 297-303, 1975.

21. Rahn, H., C. V. PAgAnelli, AND A. AR. The avian egg: aircell gas tension, metabolism and incubation time. Respir. Physiol. 22: 297309, 1974.

22. Romanoff, A. L. Pathogenesis of the Avian Embryo. New York: Wiley, 1972.

23. Shuttleworth, T. J. Fluoroaluminate activation of different components of the calcium signal in an exocrine cell. Biochem. J. 269: 417-422, 1990.

24. TASHIAN, R. The carbonic anhydrases: widening perspectives on their evolution, expression and function. Bioessays 10: 186-192, 1989.

25. Tazawa, $\mathrm{H}$. Oxygen and $\mathrm{CO}_{2}$ exchange and acid-base regulation in the avian embryo. Am. J. Zool. 20: 395-404, 1980.

26. UI, M. Islet-activating protein, pertussis toxin: a probe for functions of the inhibitory guanine nucleotide regulatory component of adenylate cyclase. Trends Pharmacol. Sci. 5: 277-299, 1984.

27. Vandecasserie, C., C. Paul, C. Schnek, and J. Leonis. Oxygen affinity of avian hemoglobins. Comp. Biochem. Physiol. A Comp. Physiol. 44: 711-718, 1973.

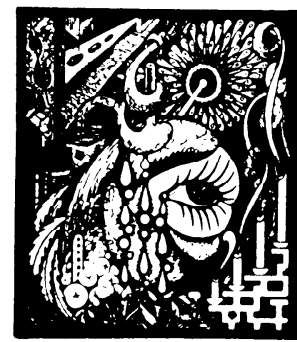

